ARTICLE

\title{
Why has the COVID-19 pandemic increased support for Universal Basic Income?
}

\author{
Daniel Nettle (i) ${ }^{1 凶}$, Elliott Johnson (i) ${ }^{2}$, Matthew Johnson ${ }^{2} \&$ Rebecca Saxe ${ }^{3}$
}

The onset of the 2020 global COVID-19 pandemic led to a marked increase in positive discussion of Universal Basic Income (UBI) in political and media circles. However, we do not know whether there was a corresponding increase in support for the policy in the public at large, or why. Here, we present three studies carried out during 2020 in UK and US samples. In study 1 ( $n=802$, April 2020), people expressed much stronger support for a UBI policy for the times of the pandemic and its aftermath than for normal times. This was largely explained by the increased importance they attached, in the pandemic context, to a system that is simple and efficient to administer, and that reduces stress and anxiety in society. In study 2 $(n=400$, May 2020), we pitted UBI against a conditional targeted social transfer system. Preferences for $\mathrm{UBI}$ were stronger for pandemic times than for normal times. This was partially explained by a number of perceived advantages, such as simplicity of administration and suitability for a changing world. In study 3 ( $n=397$, September 2020), we found that the headline results of studies 1 and 2 persisted six months after the onset of the pandemic, albeit with attenuated effect sizes. Our results illustrate how a changing social and economic situation can bring about markedly different policy preferences, through changes in citizens' perceptions of what is currently important.

\footnotetext{
${ }^{1}$ Newcastle University Population Health Sciences Institute, Newcastle University, Newcastle, UK. ${ }^{2}$ Politics, Philosophy and Religion, Lancaster University, Lancaster, UK. ${ }^{3}$ Brain and Cognitive Sciences and McGovern Institute for Brain Research, Massachusetts Institute of Technology, Cambridge, MA, USA.

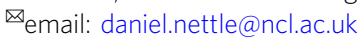




\section{Introduction}

he global COVID-19 pandemic of 2020 appears to have led to a marked increase in discussion of Universal Basic Income (UBI) as a major plank of public policy. UBI is a regular payment made to individuals by the state. Although exact definitions vary, the recurrent constitutive features are very broad coverage (for example, every citizen); and minimal or absent conditionality (Francese and Prady, 2018). Thus, UBI provides a guaranteed minimum income regardless of events and is not withdrawn if individuals receive other payments. Arguments for UBI, based both on its potentially positive social and economic effects and on fundamental ethical principles, have existed for many years (see Standing, 2017; van Parijs and Vanderbroght, 2017). In 2020, a number of academic and media articles have appeared specifically citing the pandemic as making these arguments more compelling (e.g., Bush, 2020; Hensher, 2020; Volpicelli, 2020). The Spanish government declared an intention to incorporate a UBI, which is now likely to be realised as a minimum income guarantee to some 2.5 million people, as a fundamental part of its policy response ( $\mathrm{Ng}, 2020)$. In the UK, a motion to introduce a UBI has been signed by 100 parliamentarians from across the political spectrum (Pickard, 2020). Thus, it would seem that the crisis has shifted the UBI debate. However, we do not currently know how general this shift might be; it could be restricted to a few policymakers and commentators. Thus, the first question we address in this study is whether the pandemic has made UBI more attractive for members of the UK and US populations in general.

If a broader shift in public opinion did exist, this would present an opportunity to expose what the appealing and unappealing features of UBI were anyway. UBI has been advocated as desirable on a range of ethical, health and economic grounds (Martinelli, 2017; Standing, 2017; van Parijs and Vanderbroght, 2017; Johnson et al., 2020). However, evidence regarding its broad public appeal is more mixed. On the one hand, recent polling evidence shows moderate or high levels of support in many developed countries. In the European Social Survey of 2016, 56\% supported the idea of UBI, with the proportion exceeding $45 \%$ in 20 of the 23 countries (Roosma and van Oorschot, 2019). Majorities of people find convincing pro-UBI arguments based on the provision of security and reduction of bureaucracy (Ipsos MORI, 2017).

On the other hand, support drops quite markedly when respondents are told that a UBI scheme would come at the expense of higher taxation or existing welfare benefits (Ipsos MORI, 2017). In the same polls that find large pluralities supportive of a UBI, pluralities of respondents also feel that there would be negative consequences for willingness to work (Dalia Research, 2017), or that there are superior ways of targeting help to those who need it most (Populus, 2018). Note that the latter is not necessarily the case: an unconditional scheme like UBI can have any distributional consequence desired when coupled appropriately with the taxation of income. For example, if an annual UBI of $€ 12,000$ were coupled with a flat income tax rate of $50 \%$, the effect of the tax and transfer system would be: to give $€ 12,000$ to those with no other income; leave unchanged the income of those earning $€ 24,000$ gross; and take away $€ 228,000$ from those earning $€ 600,000$ gross (Straubhaar, 2017). This redistributive consequence may not be intuitively obvious, given that the richest in society would still receive their UBI payments. There may be a conflation in people's minds between targeting, which is channelling resources to people who need them most, and conditionality, the requirement to demonstrate need in each individual case (on deservingness and the requirement to demonstrate it as intuitive principles underlying support for welfare systems, see Stone, 1984; Aaroe and Petersen, 2014; Piff et al., 2020).
One account of the apparently contradictory findings on public support for UBI is that people can infer and represent a range of both negative and positive features of the proposed policy, often simultaneously. Their overall support will reflect the weights they assign to these negative and positive features. The weights a person assigns could respond to features of the current situation, as Nettle and Saxe (2020) recently argued. Thus, a policy that feels right for peacetime may not feel suitable for war; and, pertinently, one that feels right for normal times may no longer feel right for a pandemic. If this broad view is right, then any pandemic-related changes in support for UBI will be at least partly explicable by shifts in the importance assigned to perceived negative and positive consequences of the policy.

Here, we report two main studies of UK and US respondents conducted during the height of pandemic lockdowns. These were not representative samples, and thus our surveys do not allow us to estimate the absolute level of current support for UBI in those countries. Rather, we were interested in within-individual alterations in view in the context of the pandemic. Unfortunately, we were not able to carry out a true longitudinal study of support for UBI as the pandemic unfolded, as we had no prepandemic baseline data. Instead, we asked participants to think about how they would have felt about the policy in normal times as if the pandemic had not happened, and how they now felt about it for the times of the pandemic and its aftermath. Hence, whenever we refer to pandemic-related shifts in support or preference, we mean self-reported differences between the times of the pandemic and its aftermath and the hypothetical case where the pandemic had not occurred.

We hypothesised that participants would see UBI as more attractive for the pandemic times than when thinking about normal times. We then explored what importance respondents assigned to commonly-discussed advantages and disadvantages of UBI. We examined how the importance of these considerations differed in the context of the pandemic, compared to normal times. Our hypothesis was that any self-reported shift in support for UBI would be systematically related to, and hence explicable by, situation-related changes in what was deemed important in a social transfer policy.

In addition to the main studies, we also conducted a shorter follow-up survey for the UK only in September 2020. This featured just the central variable of overall support for UBI for pandemic and normal times. By this time, six months into the pandemic, the strictest lockdown measures had been eased, though some restrictions were still in force, and the social and economic impact was still ongoing. The aim of the follow-up was to understand whether self-reported shifts in support for UBI in the context of the pandemic could be replicated and whether they had attenuated or strengthened over time.

\section{Study 1}

In study 1, our key outcome measure was how good an idea the respondent thought a UBI scheme, which we described to them in brief, would be for their country. Each respondent answered twice: once thinking about how things were in normal times as if the pandemic had not occurred, and once thinking about the times of the pandemic and the aftermath. We also listed a set of nine propositions about UBI that might be considered advantages (e.g., simplicity to administer) or disadvantages (e.g., receipt by rich people who do not need it). The propositions were generated through discussion amongst the authors, on the basis of our understanding of commonly discussed reasons for and against the policy (e.g., Gibson et al., 2018). Respondents rated the importance of each proposition, again twice, for normal and pandemic times. 
Table 1 Sociodemographic statistics for the respondent samples for study 1.

\begin{tabular}{llll} 
& UK $(\boldsymbol{n}=\mathbf{4 0 0})$ & USA $(\boldsymbol{n}=\mathbf{4 0 2})$ & \multicolumn{1}{c}{ Overall $(\boldsymbol{n}=\mathbf{8 0 2})$} \\
\hline Age & $36.35,12.66,18-82$ & $30.76,10.18,18-69$ & $33.55,11.81,18-82$ \\
Gender & Male 199, Female 201 & Male 200, Female 202 & Male 399, Female 403 \\
Subjective socioeconomic status (ladder position, $1=$ bottom, $10=$ top) & $5.19,1.69,2-10$ & $5.22,1.80,1-10$ & $5.21,1.74,1-10$ \\
Political orientation $(0=$ left, $100=$ right) & $42.68,21.62,0-100$ & $34.82,25.61,0-100$ & $38.75,24.01,0-100$ \\
Personally affected by pandemic $(0=$ not at all, $100=$ very much) & $58.91,25.06,0-100$ & $56.52,26.75,0-100$ & $57.71,25.93,0-100$ \\
\hline Shown are means, standard deviations, and ranges; or counts. & & \\
\hline
\end{tabular}

We aimed to predict a respondent's support for UBI by their rated importance of the advantages and disadvantages. We then asked: what propositions does the pandemic make more important, and what propositions less important? Hence, can we explain why support for UBI is shifted by the pandemic, assuming that it is?

Data were gathered on April 7th, 2020, from members of prolific.co, a crowd-sourcing platform for psychological and social research. By that date, in the UK, there had been 55,000 positively identified cases and 7500 deaths from COVID-19 (Financial Times, 2020). Strict 'stay at home' regulations had been in place since March $23^{\text {rd }}$. In the USA, almost 400,000 cases and 13,000 deaths had been identified (Financial Times, 2020). Most states had introduced 'stay at home' regulations over the three weeks prior to data collection.

\section{Methods}

Participants. 802 members of prolific.co completed the study

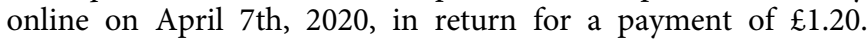
Responses were limited to those both born and resident in either the UK or USA, with equal target sample sizes for each. Samples in each country were gender-balanced by design. The study (along with studies 2 and 3) was approved by the Faculty of Medical Sciences ethics committee, Newcastle University.

Design and measures. Our materials and analysis plan were preregistered and are freely available at: https://osf.io/eyq92/ (this includes the full wording of the survey). The survey first described a UBI as: "a social security system where every citizen is paid a modest guaranteed income every month, just enough to cover basic necessities. The payment is the same for everyone. The payment is not conditional on what other earnings the person has and they do not have to do anything in particular to receive it". Respondents were then asked, thinking about normal times as if the current pandemic had not occurred, how bad or good an idea it would be to introduce a UBI in their country. Response was via a horizontal slider anchored with $0=$ bad idea, and $100=$ good idea. They were then asked, "thinking about how things are right now, how bad or good an idea would it be to introduce a UBI in your country for the period of the current COVID-19 pandemic and its aftermath?" Response was again via a horizontal slider from 0 to 100 .

A second section of the survey listed, in random order, nine propositions expressing advantages or disadvantages of UBI (for wording see protocol). For each proposition, participants were asked to rate first how true they it was of UBI; second, how important they saw it being for the desirability of UBI in normal times; and third, how important they saw it being for the desirability of UBI in the times of the pandemic and its aftermath, again on horizontal sliders from 0 to 100 . All nine propositions were rated as true of UBI to a substantial extent (mean truth ratings 43.12-75.97). This satisfied us that we had identified propositions that were thought of by many people as relevant advantages or disadvantages of UBI. Henceforth, we focused our analysis on the ratings of importance. Weighting the importance by the degree the proposition was thought to be true produced extremely similar results to those presented below.
We also gathered a number of sociodemographic variables: age; gender; subjective socioeconomic status via the MacArthur ladder (Operario et al., 2004); left-right political orientation; and the extent to their own life had been negatively affected by the pandemic. In addition, the survey contained several other exploratory questions regarding perceptions of the pandemic, and the likely impact of UBI on their own behaviour, that we do not analyse here (for full details see protocol).

Data analysis. Data were analysed in $\mathrm{R}$ ( $\mathrm{R}$ Core Development Team, 2018), using paired $t$-tests and general linear models, as outlined in the results section. The distribution of residuals for all models was satisfactory. All $p$-values are two-sided. Our main pre-registered confirmatory prediction was that support for UBI for pandemic times should be higher than for normal times. The rest of the analyses are considered exploratory.

\section{Results}

Characterization of sample and socio-demographics of UBI support. Table 1 provides descriptive statistics for the samples in study 1 . The samples showed a good spread of ages and a range of subjective socioeconomic statuses. Normal times support for UBI was significantly predicted by subjective socioeconomic status (with lower status entailing higher support: $B=-1.16$, s.e. 0.55 , $t=2.11, p=0.03$ ) and left-right political orientation (with more left-wing orientation entailing higher support: $B=-0.50$, s.e. $0.04, t=-12.37, p<0.001)$. Neither age $(B=-0.07$, s.e. $0.08, t=$ $-0.88, p=0.38)$ nor gender $\left(B_{\text {male }}=0.74\right.$, s.e. $1.92, t=0.39$, $p=0.70)$ were significant predictors. These sociodemographic variables only explained $17 \%$ of the variation in normal times UBI support (a figure that was not increased by adding the country to this model, and the country was not a significant predictor; $B_{\mathrm{USA}}=-2.89$, s.e. $1.99, t=-1.45, p=0.15$ ).

UBI support for pandemic versus normal times. Support for UBI was significantly higher for pandemic times (mean 80.27, s.d. 24.89) than normal times (mean 64.23, s.d. 29.81; $t=22.07$, $p<0.001$; Fig. 1A). This would be considered a large effect (Cohen's $d 0.78$ ) by conventional criteria. Of the 802 respondents, 576 supported UBI more strongly for a pandemic than normal times; 163 supported it equally; and 63 supported it less strongly. The shifts were very similar in the two countries (UK: Cohen's $d$ $0.81, t=16.21, p<0.001$; USA: Cohen's $d 0.74, t=14.94$, $p<0.001)$. Political orientation weakly predicted the magnitude of pandemic-related shifts in support, with more right-wing people having larger positive shifts $(B=0.11$, s.e. $0.03, t=2.64$, $p<0.001)$. However, this was an artefact of more right-wing people having lower support in normal times, and hence more scope for an increase (Fig. 1B). Neither UBI support in pandemic times $(B=0.03$, s.e. $0.03, t=0.78, p=0.44)$ nor the shift in support ( $B=0.00$, s.e. $0.03, t=0.07, p=0.95$ ), was predicted by the extent the respondent's own life had been negatively affected by the pandemic. 
A

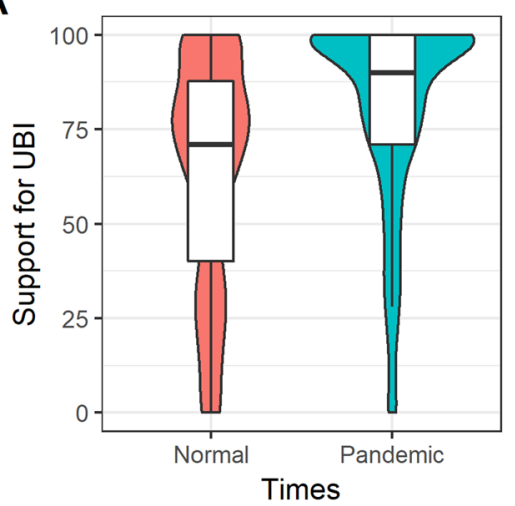

B

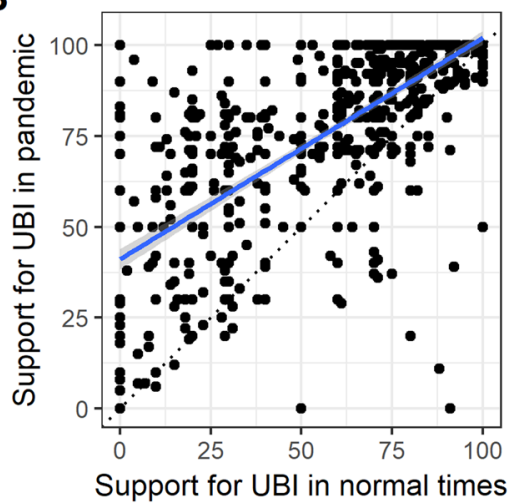

Fig. 1 Support for UBI in study 1. A Boxplots of UBI support in normal and in pandemic times. Violins show the distribution of the data. B Individual responses for UBI support in normal and pandemic times. The dotted line shows the line of equality, whilst the solid line is a linear fit through the data.

A

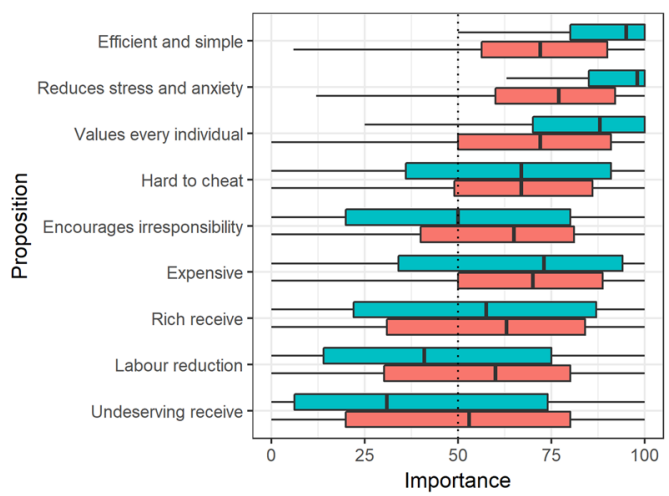

B

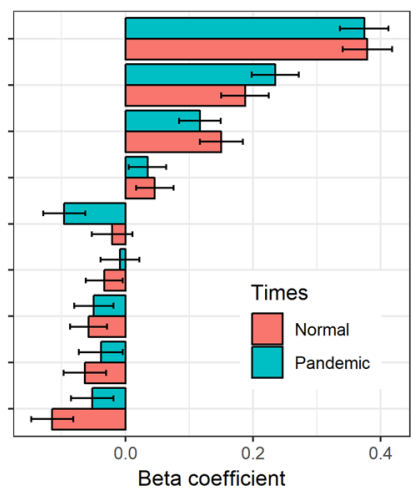

c

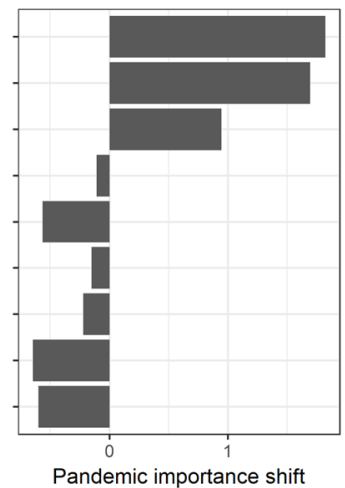

Fig. 2 Reasons for supporting UBI, study 1. A Boxplots of the rated importance of the UBI-related propositions, for normal (red) and pandemic (green) times. B Regression coefficients (standardised betas) predicting support for UBI from rated importance of nine UBI-related propositions. The model was estimated separately for the normal-times data (red), and the pandemic-times data (green). Error bars show \pm 1 standard errors. B Shift in the importance of each proposition when comparing pandemic times to normal times. Shift is expressed in terms of Cohen's $d$ (mean shift expressed in units of its standard deviation).

Predicting support for UBI from advantages and disadvantages. The importance ratings of the nine propositions relating to UBI varied substantially from individual to individual, with no proposition universally important or unimportant, especially in normal times (Fig. 2A). The normal-times importance ratings of the nine propositions together explained $49 \%$ of the variation in normal-times UBI support. The regression coefficients for each of the nine propositions are shown in Fig. 2B. Respondents supported UBI more strongly in normal times if they rated it as more important to have a system that is efficient and simple to administer; that reduces stress and anxiety; and that gives every individual a value; and, to a lesser extent, having a system that is hard to cheat. They supported UBI less strongly if they assigned more importance to labour reduction or receipt by the rich or undeserving. The remaining propositions were negligible predictors of support for UBI. We repeated the same exercise, but predicting pandemic times support for UBI from the rated importance of the nine propositions for pandemic times. The beta coefficients were virtually identical to those for normal times (Fig. $2 \mathrm{~B}$; variance explained $45 \%$ ).

Explaining the shift in support for UBI with the pandemic. We calculated the standardised shift in rated importance of each proposition from normal to pandemic times (Fig. 2C; all these shifts were significantly different from zero at $p<0.05$, apart from 'hard to cheat'). Efficiency and simplicity to administer; reduction in stress and anxiety; and giving every individual a value all became substantially more important. By contrast, labour reduction, and receipt by the rich and undeserving, all became somewhat less important. Those propositions that became more important in the pandemic are exactly those that positively predicted support for UBI in normal times, whilst those that became less important tended to be negative predictors (compare Fig. 2B, C).

We combined the normal-times regression model of UBI support on the rated importance of the propositions with the observed shifts in the rated mean importance of each proposition as we move to pandemic times. This predicted that UBI support should increase by a mean of 15.73 points for pandemic times, remarkably close to the observed mean shift of 16.03 points. We then created a predictor variable for each respondent's personal shift in support, using the betweenindividuals regression model of how normal-times UBI support relates to importance ratings, in conjunction with that individual's personality changes in importance ratings. This predictor variable, in interaction with normal-times support, did indeed significantly predict individuals' actual shifts (main effect: $B=-0.54$, s.e. $0.07, t=8.02, p<0.001$; interaction: $B=0.004$, s.e. $0.001, t=-3.02, p<0.001)$. It explained an additional $7 \%$ of the variation compared to normal-times support alone. 
Discussion. We found substantially higher support for the introduction of UBI for the times of the pandemic and its aftermath than normal times. Moreover, people's support for UBI was systematically related to what they viewed as important in a social transfer policy: the more important they rated simplicity and efficiency of administration, reducing stress and anxiety, giving every individual a value, and having a system that is hard to cheat, the more they supported UBI. Conversely, the more important they considered possible labour market reduction, and receipt by the rich and undeserving, the less they supported UBI. The pandemic did not change this pattern of associations. It merely shifted the level of importance of the different considerations: efficiency and simplicity of administration, and reducing stress and anxiety, in particular, became substantially more important to many respondents; and hence, it is logical that their degree of support for UBI increased concomitantly. We found, in general, a close correspondence between the observed shift in support for UBI, and the shift we ought to have expected given the shifts in what people viewed as important under the pandemic situation.

We found a high level of support for UBI in these samples: even for normal times, the mean support was 64 on a 100-point scale. UBI was most supported by those who viewed themselves as left-wing, and somewhat more by those whose subjective socioeconomic status was lower, but there were no significant age or gender differences. As these are features of study 2 as well, we return to them in the General Discussion. We also defer discussion of what the most important advantages and disadvantages of UBI were perceived to be until that point.

There have been many media discussions of the desirability of a UBI-style policy for the pandemic situation and its aftermath (see "Introduction" section). Whether our respondents had encountered these and been convinced by them or shifted their opinions independently, we are unable to say. However, the data do show that the case for a UBI-style response to the pandemic appears to be compelling to many people in these samples, and to a very similar degree in the two countries. We found a high degree of cognitive coherence in people's views on UBI: they could represent both bad and good features, and their overall support for the policy was fairly well predicted by the weights they assigned to those features. The pandemic situation changed those weights, and hence changed the overall support for the policy in an explicable way.

\section{Study 2}

Though study 1 revealed higher support for UBI for pandemic times, it suffered from a major limitation. Participants were not presented with an alternative to UBI that they might favour even more strongly. What we detected may not have been a pandemicrelated increase in support for UBI specifically, but a pandemicrelated increase in support for social transfer schemes in general, with UBI being the scheme we happened to mention. Indeed, past research has found that those who hold a more favourable view of UBI often also hold favourable views of conditional social transfer schemes (Roosma and van Oorschot, 2019). In study 2, therefore, we investigated how the pandemic affected people's preference between a UBI scheme and a conditional scheme, where these were mutually exclusive alternatives. In constructing our survey, we stipulated that the two schemes would cost the same amount of money overall, to remove any confound from respondents merely wishing more money to be spent on social transfers.

The design of study 2 was analogous to study 1 . Participants expressed a preference, on a continuum, between a brieflydescribed conditional targeted scheme and a briefly-described UBI scheme. We dubbed the non-UBI alternative a targeted welfare scheme', but we were explicit in the description that we meant a scheme where there were conditional criteria for receiving assistance. As we were trying to evoke intuitions about conditionality in general, rather than any particular instantiation of it, we did not specify the exact nature of the conditionality. Instead, we gave the examples of low income or inability to work and specified that citizens would have to apply for the scheme and have their eligibility assessed (see protocol at https://osf.io/ ud4v2/ for exact wording).

Participants expressed their preference once for normal times, and again for the times of the pandemic and its aftermath. In addition, we presented a set of sixteen desirable properties of social transfer systems. This set was enriched compared to the nine propositions of study 1 , to include broader considerations such as likely effects on crime and the economy and the extent to which the respondent thought they might benefit personally. Our goals were to examine which properties respondents felt were better satisfied by a UBI and which by a targeted system; and how important each property was, for normal times and for pandemic times. Hence we sought to explain why any pandemic-related shift from a preference for targeting to UBI had occurred, in terms of situation-related shifts in what properties are important.

In addition to the above, we included in study 2 some additional questions designed to shed further light on the findings of study 1 . Specifically, in relation to the study 1 finding that people think the pandemic has made it more important to reduce stress and anxiety, we asked how much respondents thought the pandemic had increased stress and anxiety, and for whom. In relation to the study 1 finding that the pandemic makes the simplicity of administration more important, we asked additional questions designed to probe why the administration might be particularly difficult in pandemic times.

\section{Methods}

Participants. As for Study 1, participants were members of prolific.co. Study 1 participants were excluded from participation. In view of the clear patterns obtained in study 1 , we reduced the sample size to 400 respondents. Again the sample was equally balanced between the UK and USA, and between genders. Participants received $£ 1.50$ for taking part. Data collection was on May 7th, 2020. By this time, the UK had over 200,000 cases and 30,000 confirmed deaths. The USA had 1.2 million confirmed cases and almost 70,000 deaths (Financial Times, 2020). Stay at home measures were still in place substantially unchanged in both countries.

Design and measures. The materials and pre-registered protocol are freely available at: https://osf.io/7qwfm/ (this includes the full wording of the survey). The survey began by briefly describing a UBI and a conditional targeted welfare system. Participants were then presented with 16 positive propositions (order of presentation randomised) representing properties that might be desirable in any social transfer system (e.g., 'Would be effective at reducing stress and anxiety'; 'Would be simple and easy to administer') and, for each, asked to provide their view on whether a targeted welfare scheme or a UBI would better satisfy the property. Responses were given via horizontal sliders from 0 'More true targeted' to 100 'More true universal' via 50 'About equally true', with the initial slider position set at 50 . The composition of this set arose from discussion amongst the authors in light of the study 1 findings.

In a second section of the survey, participants were asked: 'Thinking about how things are in normal times if the current pandemic had not occurred, do you think a targeted welfare system or a Universal Basic Income system is a better idea, 
Table 2 Sociodemographic statistics for the respondent samples for study 2.

\begin{tabular}{llll} 
& UK $(\boldsymbol{n}=\mathbf{2 0 0})$ & USA $(\boldsymbol{n}=\mathbf{2 0 0})$ & Overall $(\boldsymbol{n}=\mathbf{4 0 0})$ \\
\hline Age & $33.98,12.54,18-75$ & $35.83,12.17,18-71$ & $34.90,12.37,18-75$ \\
Gender & Male 98, Female 101, & Male 98, Female 100, Other & Male 196, Female 201, Other \\
& Other 1 & 1, PNTS 1 & 2, PNTS 1 \\
Subjective socioeconomic status (ladder position, & $5.22,1.71,1-9$ & $5.40,1.64,1-9$ & $5.68,1-9$ \\
$\begin{array}{l}1=\text { bottom, } 10=\text { top) } \\
\text { Political orientation }(0=\text { left, } 100=\text { right) }\end{array}$ & $39.22,22.39,0-100$ & $34.77,25.33,0-100$ & $38.99,23.89,0-100$ \\
Personally affected by pandemic (0 = not at all, & $58.79,24.43,0-100$ & $53.44,26.95,0-100$ & $56.11,25.83,0-100$ \\
$100=$ very much) & & \\
\hline Shown are means, standard deviations, and ranges; or counts. & & \\
\hline
\end{tabular}

assuming the two systems cost exactly the same amount of money over all?'. The following question was: 'Now thinking about the times of the current pandemic and its aftermath, do you think a targeted welfare system or a Universal Basic Income system is a better idea, assuming the two systems cost exactly the same amount of money over all?'.

A subsequent section repeated the 16 propositions (again in random order) twice more, asking for the respondent's view on how important this property would be of any social transfer system (using a slider from 0 'Very unimportant' to 100 'Very important'). In the first block, they were asked to think about normal times and in the second block, pandemic times. The propositions were rephrased declaratively for this section (e.g 'It is effective at reducing stress and anxiety'; 'It is simple and easy to administer').

A penultimate section asked five questions designed to further understand the findings concerning stress and anxiety, and simplicity of administration, from study 1 . Specifically, we asked how much the respondent thought the pandemic had increased stress and anxiety first, for those already receiving welfare payments; and second, for those who were not previously receiving welfare payments. We also asked whether the pandemic had made it more difficult than before for the government to accurately assess people's need; whether the pandemic made it more exhausting and stressful for people to demonstrate their need; and whether the desperation caused by the pandemic meant that people could not now afford the delays caused by extra paperwork.

The final section asked for the same sociodemographic variables as in study 1 . In addition to the single-item left-right political orientation measure, we also used two further items to separate social from economic conservatism (Claessens et al., 2020; see protocol for wording). Although the correlation between social and economic conservatism was only modest $(r=0.27)$, both measures correlated in an expected way with the single-item left-right measure (economic conservatism: $r=$ -0.53 ; social conservatism: $r=-0.50)$. Moreover, they did not show differential associations with UBI preference. Hence, in what follows, we restrict attention to the single-item left-right measure.

Data analysis. Data were analysed in $\mathrm{R}$ ( $\mathrm{R}$ Core Development Team, 2018) using t-tests and general linear models, as outlined below. The distribution of residuals for all models was satisfactory. All p-values are two-sided. Our data analysis strategy essentially mirrored that for study 1 , with the difference that the outcome variable is (shift in) system preference rather than UBI support; and in study 2 there were sixteen desirable properties, rather than nine propositions, as predictors. Our pre-registered predictions were that the pandemic would be associated with a shift in preference away from a targeted system, and towards UBI; and that this shift would occur across the political spectrum. All other analyses were described as exploratory. The property of not discouraging work was not asked for pandemic times, due to human error. This variable is therefore excluded from analyses involving pandemic times or the shift from normal to pandemic times.

\section{Results}

Characterization of sample and socio-demographics of UBI support. Table 2 provides descriptive statistics for the samples in study 2. The distributions of ages, subjective socioeconomic status, political orientations, and extent affected by the pandemic were very similar to study 1 , and similar across the two countries. Amongst the sociodemographic variables, system preference was predicted by political orientation (with more left-wing orientation entailing higher, i.e., more pro-UBI, system preference: $B=$ -0.46 , s.e. $0.07, t=-6.56, p<0.001)$. Neither age $(B=-0.05$, s.e. $0.13, t=-0.39, p=0.70)$, gender $\left(B_{\text {male }}=6.21\right.$, s.e. $3.33, t=1.86$, $p=0.06)$, nor subjective socioeconomic status $(B=-0.46$, s.e. $0.97, t=0.48, p=0.63)$ were significant predictors. Sociodemographic variables only explained $10 \%$ of the variation in normal times system preference (a figure that was not increased by adding the country to this model, and the country was not a significant predictor; $B_{\mathrm{usa}}=2.34$, s.e. $3.27, t=0.72, p=0.48$ ).

System preference for pandemic versus normal times. System preference was significantly more pro-UBI for pandemic times (mean 70.23, s.d. 30.30) than for normal times (mean 60.35, s.d. 33.83; $t=6.86, p<0.001$; Fig. 3). This corresponds to a medium effect size (Cohen's $d=0.34$ ). Of the 400 participants, 192 had a more pro-UBI preference under pandemic than normal times; 124 had exactly the same preference; and 84 had a more pro-targeting preference. The shifts to UBI were very similar in the UK (Cohen's $d 0.35, t=5.02, p<0.001$ ) and US respondents (Cohen's $d 0.33$, $t=4.70, p<0.001)$. The shift in preference was not significantly predicted by left-right orientation $(B=-0.03$, s.e. $0.06, t=0.48$, $p=0.63)$. Neither the preference shift $(B=0.01$, s.e. $0.06, t=0.19$, $p=0.85)$ nor the absolute system preference for pandemic times $(B=-0.03$, s.e. $0.06, t=-0.57, p=0.57)$, was associated with the extent to which the respondent's own life had been affected by the pandemic.

Which desirable properties are better served by a targeted system, and which by UBI?. All of the sixteen desirable properties had mean system ratings that were significantly different from 50 (one-sample $t$-tests, all $p<0.001$; Fig. 4). Fifteen of the sixteen were on average rated as better delivered by UBI. The only one better delivered by a targeted system was making sure that help goes to those most in need. The effect sizes of the UBI advantage were variable, with UBI most clearly preferable for simplicity of administration, suitability for an unpredictable world, being hard 
A

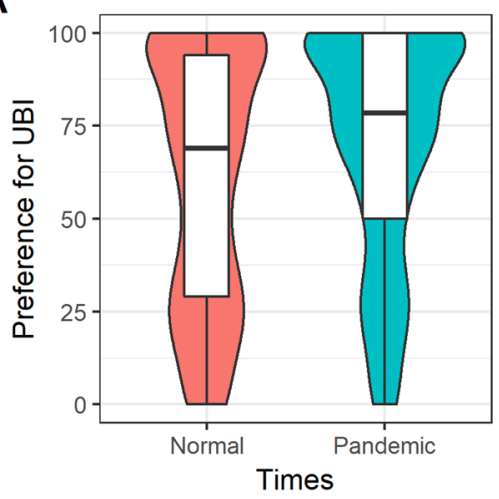

B

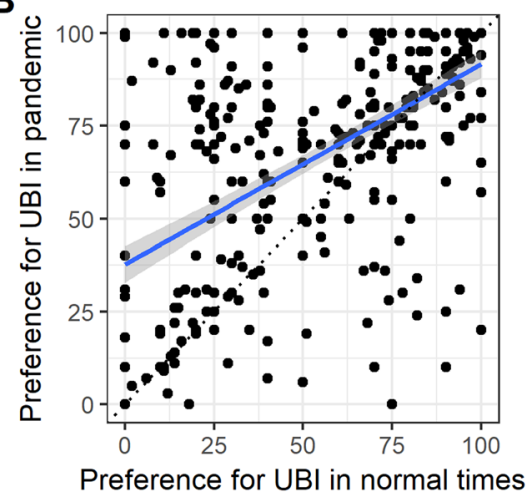

Fig. 3 Support for UBI in study 2. A. Boxplots of preference for UBI versus a targeted system in normal and in pandemic times. Violins show the distribution of the data. B. Individual responses for preference for UBI versus a targeted system in normal and pandemic times. The dotted line shows the line of equality, whilst the solid line is a linear fit through the data.

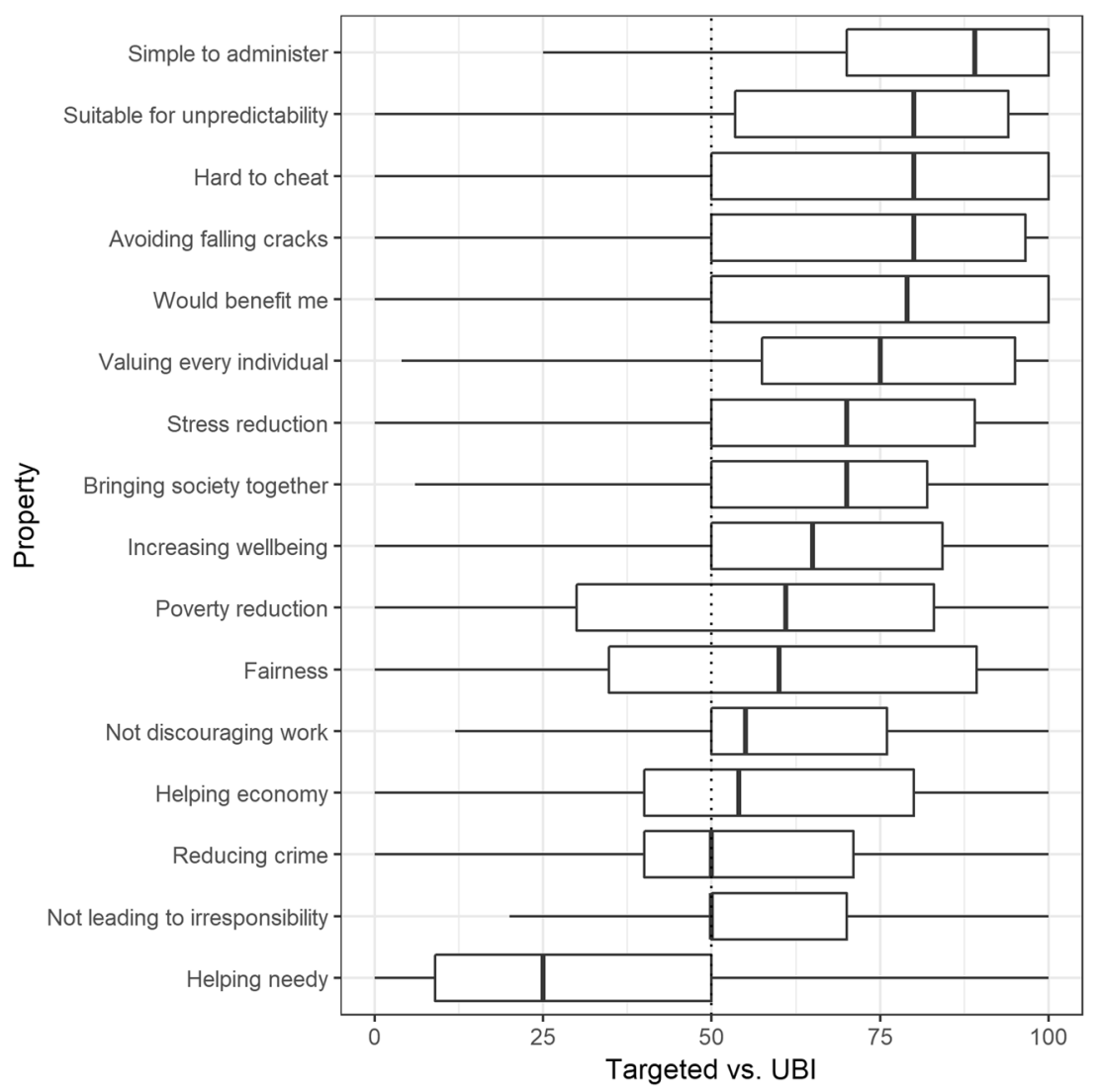

Fig. 4 Advantages and disadvantages of UBI, study 2. Boxplots of ratings of the extent to which a targeted welfare system (0) or a UBI (100) better satisfies sixteen properties of a social transfer system.

to cheat, avoiding people falling between the cracks, and benefiting the respondent personally (Fig. 4).

Predicting system preference from the importance of properties. All sixteen of the properties were on average considered moderately to highly important, but each showed considerable variation, with different properties most important to different people (Fig. 5A). We created a general linear model of system preference in normal times from the rated normal-times importance of the sixteen properties. This model explained $18 \%$ of the variation. The regression coefficients from this model are shown in Fig. 5B. The strongest positive predictors were simplicity of administration, poverty reduction, suitability for unpredictability, and personal benefit. The strongest negative predictors were making sure help was directed to the neediest, having a system that was hard to cheat, and not leading to irresponsible behaviour. We repeated the same exercise for pandemic times (Fig. 5B). The variance explained by this model was $11 \%$. The observed relationships showed some similarities to, and some discrepancies from, normal times. For example, attaching importance to the simplicity of administration was a positive predictor of UBI preference in both normal and pandemic times. By contrast, attaching importance to a system that would personally benefit the respondent was a positive predictor of UBI preference in normal times, but tended 
A

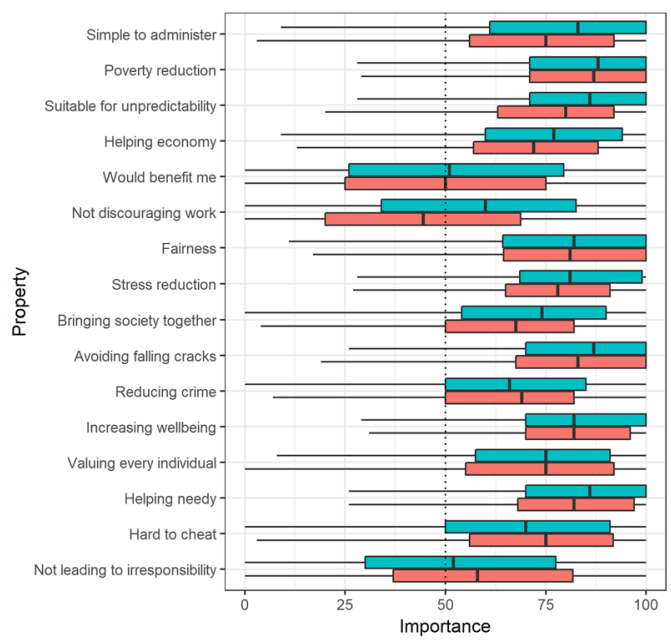

B

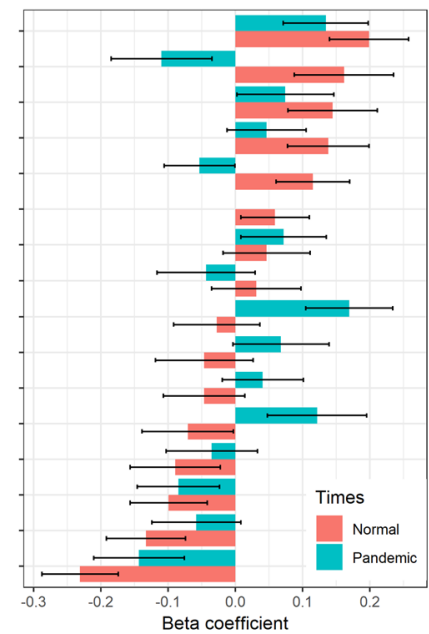

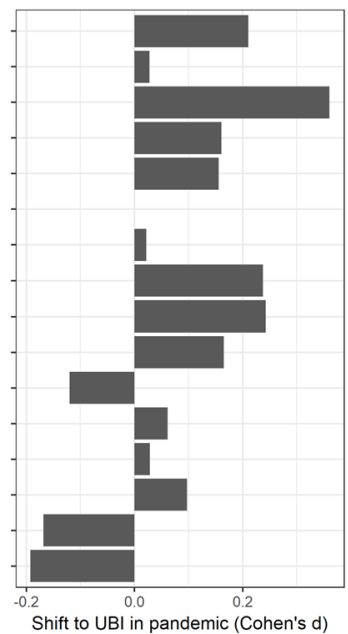

Fig. 5 Reasons for supporting UBI, study 2. A Boxplots of rated importance of sixteen properties of a social transfer system, for normal and pandemic times. B Regression coefficients (standardised betas) predicting preference for UBI from rated importance of the sixteen properties. The model was estimated separately for the normal-times data (red), and the pandemic-times data (green). Error bars show \pm 1 standard errors. C Shift in importance of each property when comparing pandemic times to normal times. Shift is expressed in terms of Cohen's $d$ (mean shift expressed in units of its standard deviation).

to be a negative one in pandemic times; and bringing society together was a positive predictor of UBI preference in pandemic times, but not in normal times.

Explaining the shift in system preference with the pandemic. The standardised shift in rated importance of each property when thinking about pandemic times is shown in Fig. 5C. All these shifts were significantly different from 0 at $p<0.05$, with the exception of poverty reduction, fairness, increasing wellbeing, valuing every individual, and helping the needy (the last was only marginally non-significant, $p=0.05$ ).

To predict the mean shift in system preference from normal to pandemic times, we combined the normal-times regression model of UBI preference on rated importance of the properties, with the observed pandemic-related importance shifts. This predicted that UBI preference should increase by a mean of 5.16 points for pandemic times. This somewhat underestimated the observed shift of 9.88 points. We then created a variable to predict each individual's shift in system preference from their individual shifts in property importance. This predictor variable, in interaction with normal-times preference, significantly predicted the shift in preference (main effect: $B=-0.38$, s.e. $0.16, t=-2.44, p=0.02$; interaction: $B=0.005$, s.e. $0.002, t=2.25, p=0.03$ ), though with only a small increase in variance explained (1\%) compared to a model including normal times preference alone.

Further questions on the consequences of the pandemic. Respondents felt that the pandemic had increased stress and anxiety for people not previously receiving welfare payments substantially more than it had for people who had previously been receiving welfare payments (not previously receiving: mean 74.81, s.d. 20.76; previously receiving: mean 55.61, 30.33; $t=10.55, p<$ $0.001)$. In a model predicting pandemic-times system preference from normal-times system preference and the answers to the two stress and anxiety questions, the extent to which the pandemic had increased stress and anxiety for people previously receiving welfare payments was not a significant predictor $(B=-0.03$, s.e. $0.04, t=-0.84, p=0.40$ ), but the extent to which it has increased stress and anxiety for those not previously receiving welfare payments was $(B=0.15$, s.e. $0.06, t=2.54, p=0.01)$. In other words, people's pandemic-related preference was more pro-UBI to the extent that they thought the pandemic had negatively affected people not previously receiving welfare payments, but unrelated to their views of its effect on those previously in receipt of welfare.

There were high levels of endorsement of the statements that the pandemic made it hard for the government to assess need (mean 71.94, s.d. 24.44); made it more exhausting and stressful for individuals to demonstrate their need (mean 76.64, 20.86); and made people so desperate that they could not afford the delays caused by paperwork before receiving assistance (mean 85.61, s.d. 16.57). Of these, the first two items did not significantly predict a more pro-UBI system preference for pandemic times $(B=-0.02$, s.e. $0.06, t=-0.37, p=0.71 ; B=0.05$, s.e. 0.07 , $t=0.72, p=0.47)$, but the third did $(B=0.23$, s.e. $0.08, t=2.85$, $p=0.005)$

Discussion. In study 2, we pitted a UBI scheme against a conditionally targeted welfare scheme that would cost the same overall. Respondents were relatively favourable to the UBI scheme in normal times, but more saliently, shifted their preference in the pro-UBI direction for pandemic times. This shift was smaller than the shift in general UBI support observed in study 1 but still substantial. Thus, only part of the increase in pandemic-related UBI support seen in study 1 represents an increase in support for generous social transfers in general. There is also an increase in attraction to universality and unconditionality in particular. This shift was observed in exactly the same way in both countries.

Compared to the conditional targeted system, UBI was favoured on average for all properties other than making sure help went to those most in need. In fact, it is not necessarily the case that a conditional system is more effective at reaching those in most need: people in need often fail to access conditional benefits due to stigma or the difficulty making claims; and coupled with an appropriate taxation scheme, the distributional effect of UBI can favour those on the lowest incomes. Our specification that the two schemes would 'cost the same over all' may not have helped here. If respondents were considering the 
gross cost, they would have inferred that those on low incomes would get less under the UBI scheme than the conditional one, but we did not ask them this and hence cannot be sure. Perhaps what our respondents were reacting to was the 'making sure' aspect: making sure implies conditional assessment in many people's minds. Alternatively, many of our respondents could have had the incorrect intuition that UBI schemes cannot have redistributive effects. The potential of UBI for helping those most in need is, therefore, a feature that advocates of the policy need to find ways of communicating since it does not seem to be intuitively obvious.

For the other properties, the degree of UBI advantage varied with the property in question, being strongest for simplicity of administration, being hard to cheat, and avoiding people falling between the cracks. These particular samples of respondents also mostly felt that a UBI scheme would benefit them personally more than a conditionally targeted scheme.

We were able to partly explain the shift in preference towards UBI for pandemic compared to normal times. The simplicity of administration, suitability for an unpredictable world, need to reduce stress and anxiety, and the need to bring society together were all rated as substantially more important for pandemic times, and these were all properties that favour UBI. By contrast, avoiding irresponsible behaviour, a negative predictor of UBI preference, was rated as less important for pandemic times. However, respondents' shifts in importance ratings did a less good job of predicting their shift in system preference, compared to study 1. The reasons for people's preferences between conditional targeted welfare and UBI appeared to be less coherent and explicable than their overall level of UBI support. There are a number of lines of evidence for this claim. First, sociodemographic variables such as left-right orientation explained only $10 \%$ of the variation in system preference between targeting and UBI, compared to $17 \%$ of the variation in overall UBI support in study 1. Second, in both studies, we obtained ratings of a set of properties of welfare systems of which the respondents rated the importance. In study 1 , these importance ratings explained fully half the variation in UBI support. In study 2, it was only 11-18\%, despite the fact that we provided respondents with a wider range of properties to rate. Third, in study 1 , the importance ratings that predicted UBI support in normal times predicted UBI support in almost exactly the same way in pandemic times, whereas in study 2 this was not true. Moreover, in study 2 we observed some puzzling discrepancies. For example, UBI was rated as substantially better than targeted welfare for being hard to cheat; and yet belief in the importance of a social transfer system being hard to cheat was a negative predictor of preference for UBI. For all these reasons, we suggest that people's intuitions about universality versus conditional targeting when presented with a direct trade-off between the two are weaker and less clear than when they are just rating a universal system against no specified alternative. Indeed, as we know, those who are more in favour of UBI also tend to be more in favour of conditional targeted welfare systems anyway (Roosma and van Oorschot, 2019), and support for UBI can be markedly decreased by pointing out explicitly that it would come at the expense of existing targeted assistance (Ipsos MORI, 2017).

A further limitation of study 2 was that in the materials of study 2 , we named the non-UBI alternative a 'targeted welfare' system. In retrospect, and as pointed out by a reviewer, the alternative scheme we described would have been better designated 'conditional' (individuals have to demonstrate eligibility via a specific assessment, as the text made clear) than 'targeted'. What study 2 measured is therefore intuitions around conditionality more than anything else
Our follow-up questions on the consequences of the pandemic shed considerable light on the findings, in both studies, that stress and anxiety reduction, and simplicity of administration, become more important in the pandemic and are central to the reasons that UBI becomes more favoured. Respondents felt that there had been an increase in stress and anxiety, but it had fallen differentially on those not previously receiving welfare payments. That is, the pandemic had brought adversity to sectors of society that were previously secure (and presumably deserved to be so). It is thence logical that augmenting the existing targeted welfare systems does not solve the problem: instead, a new form of social transfer is needed that reduces stress and anxiety for everyone. Likewise, respondents believed that the pandemic made it more difficult for governments to assess, and people to demonstrate, need; and that delays due to paperwork would be particularly unacceptable in these desperate times. This squares with the increase in the importance assigned to the simplicity of administration, which is considered one of the main strengths favouring UBI in both studies.

\section{Study 3}

The headline results of studies 1 and 2 were that people expressed stronger support for UBI for the context of the pandemic and its aftermath than for normal times. Both surveys were carried out in the first few months of the pandemic impact when strict lockdowns were in force. We were interested to understand whether the proUBI shifts in opinion were transitory, or would still be found six months into the pandemic impact. In September 2020, we, therefore, repeated just the key measures of the first two studies (support/preference for UBI for pandemic times and normal times, along with basic demographic information) in further samples of UK participants from the same participant pool. The aim of study 3 was to establish whether the pro-UBI shifts for pandemic times were still detectable and whether the effect sizes had attenuated compared to the effect sizes we found in April and May.

\section{Methods}

Participants, design and measures. As for studies 1 and 2, participants were members of prolific.co. They were not the same individuals who had taken part in studies 1 and 2. Sampling for study 3 was restricted to the UK only. We created abbreviated versions of the study 1 survey (henceforth study 3a) and the study 2 survey (study $3 b$ ). These contained basic demographic information plus: overall support for UBI for normal and pandemic times (study 3a); and degree of preference between a targeted system and UBI (study 3b). Participants ( $n=200$ for study 3a; $n=197$ for study $3 \mathrm{~b}$ ) completed the surveys on September 15 and 162020 and received $£ 0.60$ for doing so.

Data analysis. Raw data and $\mathrm{R}$ scripts are available at: https://osf. io/jwhva/. We first performed paired t-tests in each sample to establish whether support for UBI was higher for pandemic times than normal times. To examine whether the pandemic-related pro-UBI shifts were smaller in study 3 than studies 1 and 2, we combined the study $3 \mathrm{a}$ and $3 \mathrm{~b}$ data with the data from their respective main studies. We then carried out $t$-tests with each individual's difference between UBI support in pandemic times and normal times as the outcome variable, and time point (April/ May or September) as the predictor.

Results. Samples were gender-balanced (study 3a: 100 male, 100 female; study 3b: 98 male, 99 female), with similar age distributions to the main studies (study 3a: mean 33.93, s.d. 11.37; study 3b: mean 36.39 , s.d. 12.28). 


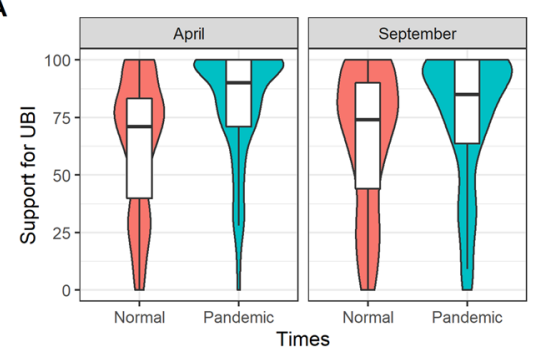

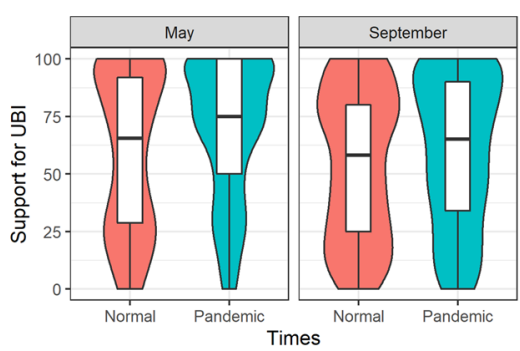

Fig. 6 Support for UBI in study 3. A Boxplots of UBI support in normal and pandemic times, study 3a (September), and the UK participants of study 1 (April). Violins show the distribution of the data. B Boxplots of preference for UBI versus a targeted system in normal and in pandemic times, study $3 b$ (September), and the UK participants of study 2 (May). Violins show the distribution of the data.

The main results are shown in Fig. 6. In study 3a, support for UBI was significantly higher for pandemic times (mean 75.05, s.d. 28.26) than normal times (mean 65.06, s.d. 29.98; $t=7.10, p<$ 0.001 ). The effect was significantly smaller for September than for the UK participants in April $(t=-3.84, p<0.001)$. The September effect size of $d=0.50$ was only $62 \%$ of the April effect size of $d=0.81$.

In study $3 b$, respondents favoured UBI significantly more strongly for pandemic times (mean 59.71, s.d. 32.20) than normal times (mean 53.93, s.d. 32.37; $t=3.48, p<0.001$ ). The effect size for September $(d=0.25)$ was $70 \%$ that found in May $(d=0.35)$. However, the difference between the two effects was not statistically significant $(t=-1.55, p=0.12)$.

Discussion. Six months into the pandemic, and around five months after the original data collection, we again found that overall support for UBI was higher for pandemic times than for normal times; and that preference between a UBI system and a conditional targeted system was more pro-UBI for pandemic times than normal times. Thus, we both replicated the headline findings of studies 1 and 2 and showed that the pro-UBI shifts have persisted beyond the immediate shock of the onset of the pandemic. However, the effect sizes had diminished: in both cases, the magnitude of the pro-UBI shift was $60-70 \%$ of what it had been in the original studies. The attenuation of the effect was related to pandemic-times levels of UBI support or preference being lower in study 3 than studies 1 and 2, rather than any difference in the normal-times support or preference.

\section{General discussion}

In fairly diverse samples of the UK and US population, we found substantially more positive attitudes to UBI in the context of the pandemic and its aftermath than in the context of normal times. There was substantially higher overall support for UBI (study 1), and a moderately greater preference for a UBI scheme over a conditionally targeted scheme that cost the same overall (study 2). These effects were extremely similar across the two countries and not substantially different across the left-right political spectrum. These findings suggest that the pro-UBI shifts in opinion that have appeared in the media and policy circles in the context of the pandemic either reflect or have led to, a broad shift in attitude to the policy in these populations. We also found that these shifts persisted, albeit somewhat attenuated, six months after the beginning of the pandemic.

Our studies also suggest that people support UBI for at least somewhat coherent reasons. They can represent a range of implications or consequences of the policy, both negative and positive, and their overall evaluation of it is partially predictable from the importance they assign to these features. What the pandemic does, in respondent's minds, is change the importance of several of the features; their shift in overall support for the policy then follows. This was much more clearly true in study 1 , where overall support for UBI was well predicted by rated importance of the policy's advantages and disadvantages, and the shift in support was well predicted by the shifts in those importance ratings. In study 2, where we pitted UBI directly against a conditional targeted system, the pattern was less clear: the importance ratings given to the various features of the policy were weaker and less consistent predictors of overall preference for the policy, and the shift in importance ratings was a poorer predictor of the shift in preference in relation to the pandemic. This suggests that people have intuitions about the desirability of universality and simplicity, and also about the importance of targeting the deserving, and that when the trade-off between these is made salient, the result is somewhat unpredictable.

A consistent finding across the two studies, perhaps unsurprisingly, is that simplicity of administration is seen as a major strength of UBI, and people who place a high value on such simplicity support the policy more strongly. In both studies, the pandemic increased the importance of such simplicity. Delving further through our follow-up questions in study 2, respondents felt that the pandemic favored simplicity because verification by the government of who was in need would be harder now; and, particularly, demonstration of need by desperate citizens would be difficult and lead to unacceptable delay. Also in study 2, respondents felt that the pandemic placed a greater value on the system being able to cope with unpredictable change and avoid people falling between the cracks, again strengths of UBI. These findings make sense: if the effects of the pandemic are to spread precariousness and unpredictably changing needs to all classes and occupations, then any solution based on carefully identifying the needy and eligible individuals risks failing on a large scale.

Relatedly, in both studies we found that the pandemic led to an increase in the importance of reducing people's stress and anxiety; and again, this was a property where UBI was rated highly. The pandemic was thought to have increased stress and anxiety mostly for people who were not previously being helped by the welfare system. That is, the effect of the pandemic is not so much to have made things even worse for those who were already in precariousness and need, but to bring people not previously at risk of that state firmly into it. Thus it makes sense that respondents would have shifted their preference towards the inclusion of the whole population into a universal social safety net, rather than making the selective safety net more generous for those already eligible for it. This helps square our findings with the previous literature on deservingness, which finds welfare schemes are popular to the extent that they are seen as benefiting those who are needy, try to reciprocate, and have been affected by things outside of their control (van Oorschot, 2006; Aaroe and Petersen, 2014; Piff et al., 2020). The deservingness literature suggests that people will have reservations about UBI, since those 
who benefit do not necessarily meet these criteria. However, if the pandemic means that anyone, at any time, and for an identifiable reason which is obviously beyond their personal control, may fall into sudden but deserving need, then a universal system seems more attractive, even if a collateral effect is that everyone else receives it too. The pandemic may have accelerated, in people's minds, the process that many pro-UBI commentators have previously attributed to automation: the bringing of greater and greater fractions of the population into a precariousness not of their making, until the point where since the nearly whole population is in this state, universality becomes the only reasonable option.

We must stress that our study concerns the public's perceptions of whether the pandemic means that UBI is now a better social policy, and their reasons why. Thus, our data shed no light on whether UBI would actually, by objective criteria, be a better policy given that the pandemic has happened, or why. Interestingly, though, there is generally a fairly good alignment between our respondents' reasoning about UBI, and the positive arguments advanced for the policy in the literature. For example, our respondents felt that a UBI scheme would be effective in reducing stress and anxiety. Reduced stress and anxiety have indeed been observed in multiple UBI-related trials and natural experiments, and these effects form a major part of the academic argument for the policy (Hensher, 2020; Johnson et al., 2020).

A limitation of our study already noted is that the samples are not nationally representative. We would thus be cautious about interpreting the absolute levels of UBI support as indicative of the state of public opinion in either country. However, this was not the goal of our study. Rather, our interest was in the shift in support associated with the pandemic, and the way this related to shifts in what respondents thought of as important. We measured these at the within-individual level (though see below). Our samples were fairly diverse in age, subjective socioeconomic status and political orientation, so it is reasonable to infer that the clear shifts we observed in our samples imply the existence of shifts at the population scale too. Moreover, the absolute levels of support that we observed for normal times were not so far above the recently observed range. For the UK, for example, polling in recent years has suggested around half or more of representative samples support or strongly support the policy (Ipsos MORI, 2017; Roosma and van Oorschot, 2019). Our observed mean normal-times support of 64 out of 100 may well lie in a plausible range for the current state of the population, especially if support has been increasing generally over the last three years. We also reconstructed some expected relationships that are thought to exist in the whole population (Roosma and van Oorschot, 2019): greater support for UBI from those on the political left, no gender difference, and greater support from those who see themselves as being in weaker socioeconomic positions (though this was significant only in study 1). Thus, we do not see the non-representative nature of our sampling as a major flaw in the study as long as the data are not used for purposes they do not suit.

Another limitation is potentially more serious. Our assessment of normal-times support was taken during the pandemic, simultaneously with the pandemic-times measures, by just asking the respondents what they would have thought had the pandemic not occurred. Of course, this is a counterfactual. A better design would involve a longitudinal panel study with genuinely separate prepandemic and peri-pandemic responses. However, we initiated this research only once the pandemic had begun. This limitation could actually lead to us underestimating the key pandemic-related shift in support. If respondents' normal-times responses were contaminated by their current high level of support due to the ongoing pandemic, then the true opinion shift they have undergone in the last six months or so maybe larger than our study implies. In any event, this is unknowable by any means available to us.
These limitations aside, our findings, as well as specifically demonstrating an increase in support for UBI as a consequence of the pandemic, contribute to a general view of political preference formation and the sources of social change. Nettle and Saxe (2020) argue that intuitive political preferences are not fixed individualdifferences variables, but are continuously generated by structured psychology that is highly responsive to situational features. In other words, the same people generate different ideas for how a society should work in wartime than peacetime, or when heterogeneous rather than homogeneous. They do so because they spontaneously represent and infer the demands and difficulties of each situation, leading them to weight possible advantages and disadvantages differently as the situation changes. The COVID-19 pandemic has changed the social and economic situation in massive ways for millions of people in the two countries we studied. It, therefore, represents a natural experiment for Nettle and Saxe's view of political cognition. It would be problematic for that view if we did not observe large pandemic-related changes in perceptions of what features of a social policy were important, and hence which policies were preferred. It would be interesting to explore what other social policies, beyond cash transfer schemes, people's views may have abruptly shifted in.

More generally, historical accounts of how societies change, particularly in regard to the expansion of social assistance and universal services, often stress the role of large exogenous events. For example, expanded welfare provision has been linked historically to the experience of war (Kasza, 2006; Obinger and Schmitt, 2019), and the influenza pandemic of 1918-1919 has been implicated in the gradual creation of universal access to health care (Breitnauer, 2019). Political change is not only driven by public opinion, but it is partly so. The kinds of shifts we observe here in relation to the COVID-19 pandemic provide some insight into how changes in popular opinion and belief may arise spontaneously from important society-wide exogenous events, and hence into how those events can often precipitate subsequent social change. That we found significantly smaller effect sizes in September when some restrictions in the UK had been eased and the shock of the threat may have been less pressing, reinforces is the view that public opinion shifts with the situation. Support for UBI or other radical reform measures may fluctuate as the situation continues to unfold.

\section{Data availability}

Raw data, code and protocols for all three studies are available via the Open Science Framework at: https://osf.io/vkchq/ (doi: 10.17605/OSF.IO/VKCHQ).

Received: 30 June 2020; Accepted: 25 February 2021; Published online: 17 March 2021

\section{References}

Aaroe L, Petersen MB (2014) Crowding out culture: Scandinavians and Americans agree on social welfare in the face of deservingness cues. J Polit 76(3):697. https://doi.org/10.1017/S002238161400019X

Breitnauer J (2019) The Spanish Flu Epidemic and its Influence on History. Pen and Sword, Barnsley

Bush S (2020) Covid-19 has changed my thinking on universal basic income. New Statesman. https://www.newstatesman.com/politics/economy/2020/04/covid19-universal-basic-income-benefits-welfare

Claessens S, Fischer K, Chaudhuri A, Sibley CG, Atkinson QD (2020) The dual evolutionary foundations of political ideology. Springer

Dalia Research (2017) The EU's Growing Support for Basic Income. https:// basicincome.org/wp-content/uploads/2017/05/DR-2017-survey.pdf

Financial Times (2020) Coronavirus tracked. https://www.ft.com/content/a26fbf7e48f8-11ea-aeb3-955839e06441 (Accessed: 1 June 2020) 
Francese M, Prady D (2018) What Is Universal Basic Income?. Finance and Development. 38-39. https://www.imf.org/external/pubs/ft/fandd/2018/12/ pdf/what-is-universal-basic-income-basics.pdf

Gibson M, Hearty W, Craig P (2018) Potential effects of universal basic income: a scoping review of evidence on impacts and study characteristics. Lancet 392: S36. https://doi.org/10.1016/s0140-6736(18)32083-x

Hensher M (2020) Covid-19, unemployment, and health: time for deeper solutions? BMJ 371:m3687. https://doi.org/10.1136/bmj.m3687

Ipsos MORI (2017) Poll Conducted for University of Bath-Institute for Policy Research. Universal Basic Income Research. https://www.ipsos.com/sites/ default/files/ct/news/documents/2017-09/omnibus-universal-basic-incometopline-2017.pdf

Johnson E, Johnson M, Webber L (2020) Measuring the health impact of Universal Basic Income as an upstream intervention: holistic trial design that captures stress reduction is essential. Evid Policy. https://doi.org/10.1332/ $174426420 \mathrm{X} 15820274674068$

Kasza GJ (2006) War and welfare policy in Japan. J Asian Stud 61:417-35. https:// doi.org/10.2307/2700296

Martinelli L (2017) Assessing the Case for a Universal Basic Income in the UK. Institute for Policy Research. http://www.bath.ac.uk/publications/assessingthe-case-for-a-universal-basic-income-in-the-uk/attachments/ basic_income_policy_brief.pdf

Nettle D, Saxe R (2020) Preferences for redistribution are sensitive to perceived luck, social homogeneity, war and scarcity. Cognition 198:104234

$\mathrm{Ng}$ K (2020) Coronavirus: Spain to become first country in Europe to roll out universal basic income. The Independent. https:/www.independent.co.uk/ news/world/europe/coronavirus-spain-universal-basic-income-europea9449336.html

Obinger H, Schmitt C (2019) World war and welfare legislation in Western Countries. J Eur Soc Policy https://doi.org/10.1177/0958928719892852

van Oorschot W (2006) Making the difference in social Europe: deservingness perceptions among citizens of European welfare states. J Eur Social Policy 16 (1):23-42. https://doi.org/10.1177/0958928706059829

Operario D, Adler NE, Williams DR (2004) Subjective social status: reliability and predictive utility for global health. Psychol Health 19(2):237-246. https://doi. org/10.1080/08870440310001638098

van Parijs P, Vanderbroght YY (2017) Basic income: a radical proposal for a free society and a sane economy. Harvard University Press, Cambridge

Pickard J (2020) More than 100 UK opposition politicians call for universal basic income after lockdown. Financial Times. https://www.ft.com/content/ 6b00fa50-d811-41cd-975b-6a8382ca6e91

Piff PK, Wiwad D, Robinson AR, Aknin LB, Mercier B, Shariff A (2020) Shifting attributions for poverty motivates opposition to inequality and enhances egalitarianism. Springer
Populus (2018) Univeral Basic Income Survey. https://www.thersa.org/globalassets/ pdfs/reports/realising-basic-income.pdf

R Core Development Team (2018) R: A Language and Environment for Statistical Computing. R Foundation for Statistical Computing, Vienna

Roosma F van Oorschot W (2019) Public opinion on basic income: mapping European support for a radical alternative for welfare provision. J Eur Soc Policy https://doi.org/10.1177/0958928719882827

Standing G (2017) Basic income: and how we can make it happen. Pelican, London Stone D (1984) The disabled state. Temple Press, Philadelphia

Straubhaar T (2017) On the economics of a universal basic income. Intereconomics 52(2):74-80. https://doi.org/10.1007/s10272-017-0649-8

Volpicelli G (2020) Can Universal Basic Income fix the coronavirus crisis?. Wired. https://www.wired.co.uk/article/can-universal-basic-income-fix-thecoronavirus-crisis

\section{Competing interests}

The authors declare no competing interests.

\section{Additional information}

Correspondence and requests for materials should be addressed to D.N.

Reprints and permission information is available at http://www.nature.com/reprints

Publisher's note Springer Nature remains neutral with regard to jurisdictional claims in published maps and institutional affiliations.

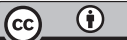

Open Access This article is licensed under a Creative Commons Attribution 4.0 International License, which permits use, sharing, adaptation, distribution and reproduction in any medium or format, as long as you give appropriate credit to the original author(s) and the source, provide a link to the Creative Commons license, and indicate if changes were made. The images or other third party material in this article are included in the article's Creative Commons license, unless indicated otherwise in a credit line to the material. If material is not included in the article's Creative Commons license and your intended use is not permitted by statutory regulation or exceeds the permitted use, you will need to obtain permission directly from the copyright holder. To view a copy of this license, visit http://creativecommons.org/ licenses/by/4.0/

(C) The Author(s) 2021 